\title{
Efecto de la Cafeína en la Motilidad y Fertilidad Espermática de Trucha Arcoiris (Oncorhynchus mykiss)
}

\author{
Nemesio I. Valdebenito \\ Universidad Católica de Temuco, Facultad de Recursos Naturales, \\ Casilla 15-D, Temuco-Chile (e.-mail: ivisler@uctemuco.cl)
}

\begin{abstract}
Resumen
En la presente investigación se evaluó el efecto en la motilidad y fertilidad espermática de trucha arcoiris (Oncorhynchus mykiss) de una solución salina 0,98\% de $\mathrm{NaCl}$ enriquecida con 2,$5 ; 5,0$; 10,0 y $20,0 \mathrm{mM}$ de cafeína, utilizando como control una solución salina sin cafeína. Al utilizar cafeína en concentraciones de $10 \mathrm{mM}$ en la solución activadora se duplicó la duración de la motilidad espermática $(165,0 \pm 18,8 \mathrm{~s})$ con respecto al control $(80,0 \pm 12,5 \mathrm{~s})$. Se registró un aumento de la fertilidad al utilizar cafeína en el activador, aunque los valores no fueron estadísticamente significativos con respecto al control $(81,6 \pm 5,7 \%)$. Estos antecedentes demuestran la capacidad de la cafeína de prolongar la motilidad espermática en trucha arcoiris, aún cuando se debe continuar con nuevos ensayos que permitan determinar las dosis mínimas de cafeína requeridas para ser utilizadas en sistemas de producción masivos.
\end{abstract}

Palabras Claves: oncorhynchus mykiss, motilidad espermática, cafeína, fertilidad en truchas

\section{Effect of Caffeine in the Motility and Fertility of Rainbow Trout (Oncorhynchus Mykiss) Sperm}

\begin{abstract}
The present investigation evaluated the effect in the motility and spermatic rainbow trout (Oncorhynchus mykiss) fertility of a saline solution $0.98 \% \mathrm{NaCl}$ enriched with $2.5 ; 5.0 ; 10.0$ and $20.0 \mathrm{mM}$ of caffeine, using as control saline solution without caffeine. The use of caffeine at a concentration of $10 \mathrm{mM}$ in the activator solution duplicated the time of spermatic motility $(160.0 \pm$ $18,8 \mathrm{~s})$ in contrast with the control $(80,0 \pm 12,5 \mathrm{~s})$. Increased fertility was observed when caffeine was used in the activator, although the values were not statistically different in relation with the control $(81.6 \pm 5.7 \%)$. These results demonstrate the capacity of caffeine to enhance spermatic motility in rainbow trout. Further research must be conducted to elucidate the minimum concentrations of caffeine required in intensive production systems.
\end{abstract}

Keywords: oncorhynchus mykiss, sperm motility, caffeine, rainbow trout fertility 


\section{INTRODUCCIÓN}

La fertilización artificial es utilizada en salmónidos desde hace varios siglos, pero el alto nivel tecnológico que en la actualidad ha alcanzado esta actividad en todo el mundo, obliga a los productores a optimizar los resultados hasta ahora obtenidos mediante el uso de activadores espermáticos con el fin de aumentar los porcentajes de fecundación. Stoss (1983) y Billard (1990), señalan que la utilización de diluyentes 0 activadores espermáticos en la fertilización artificial de salmónidos, mejora la motilidad con efectos favorables en las tasas de fertilización. Los solutos utilizados en la elaboración de los activadores espermáticos, permiten reducir el choque osmótico que sufre el espermatozoide en su contacto con el agua, le aportan energía y ajustan el pH (Ingram, 1985 y Billard, 1990). En trabajos relacionados con fecundación "in vitro" en mamíferos y seres humanos, se ha utilizado ampliamente en los activadores de motilidad espermática la epinefrina, hipotaurina y cafeína. Pocos antecedentes existen en la literatura del uso de estas sustancias con semen de salmónidos. Terner (1986) informa de la capacidad de la cafeína para intensificar la motilidad espermática en salmones, pero sin entregar mayores antecedentes sobre la técnica de utilización y su efecto en la fertilidad. Caylor et al. (1994), utilizan teofilina durante la fertilización artificial con semen criopreservado en Rachycentron canadum, pero no entrega antecedentes del efecto en la motilidad y fertilidad espermática. Valdebenito y Ratto (1996) determinan una alta correlación entre la concentración de cafeína del activador y la fertilidad en el semen de trucha arcoiris. Ciereszko et al. (1996) y Robles et al. (2003) utilizan metilxantinas para mejorar el uso del semen criopreservado de esturión y trucha arcoiris, respectivamente.

El objetivo de la presente investigación es evaluar el efecto de diferentes concentraciones de cafeína sobre la motilidad y fertilidad espermática en trucha arcoiris al ser incorporada en el activador espermático utilizado para la fertilización artificial.

\section{MATERIALES Y MÉTODOS}

Los especímenes utilizados fueron 5 machos adultos de trucha arcoiris (Oncorhynchus mykiss) cultivados en la Estación
Experimental Piscicultura Los Laureles de la Universidad Católica de Temuco, ubicada a 39/02' Lat. S y 72/12' Long W. Luego de determinar su madurez plena, los especímenes fueron anestesiados con MS222 y mediante una cánula de $2,3 \mathrm{~mm}$ de espesor, se les extrajo una muestra de semen en la que se evaluó la motilidad según la escala de Sánchez-Rodríguez y Billard (1977) que utiliza el valor "0" cuando todos los espermatozoides se encuentran inmóviles y un valor " 5 " cuando se registra una actividad flagelar intensa en todos los espermatozoides. Con los individuos en que se observó una motilidad igual a 5 , se realizó un pool que fue homogenizado suavemente y utilizando bolsas plásticas insufladas con oxígeno gaseoso, fueron trasladadas con temperaturas de entre 2 y $4^{\circ} \mathrm{C}$ y en ausencia de luz al laboratorio de reproducción ubicado a $70 \mathrm{~km}$ de la Estación Experimental. Para activar la motilidad espermática se utilizó una solución salina $(0,98 \% \mathrm{NaCl})$ preparada con agua destilada (Control). En base a ésta, se preparó cuatro soluciones que contenían cafeína en la siguientes concentraciones: 2,5mM (Tratamiento 1, $\mathrm{T}_{1}$ ); 5,0mM $\left(\mathrm{T}_{2}\right)$; $10,0 \mathrm{mM}\left(\mathrm{T}_{3}\right)$ y $20,0 \mathrm{mM}\left(\mathrm{T}_{4}\right)$. Todas las soluciones registraron un $\mathrm{pH}=9$. Veinte alícuotas de semen de $1 \mu \mathrm{L}$ fueron mezcladas con igual volumen de cada uno de los activadores y se cronometró el tiempo en que todos los espermatozoides de un área de $0,1 \mathrm{~mm}^{2}$ quedaron inmóviles. Esta experiencia se realizó a temperatura ambiente de aproximadamente $10^{\circ} \mathrm{C}$.

Para determinar el efecto de la cafeína en la fertilidad del espematozoide, se realizó un pool de ovas obtenidas de tres hembras de segundo desove de trucha arcoiris cultivadas en la misma Estación Experimental. El pool de ovas se trasladó al laboratorio utilizando la misma técnica que para el traslado de semen. Las ovas fueron separadas en 15 grupos de 300 ovas. El líquido celómico fue reemplazado por $20 \mathrm{ml}$ de los activadores de cada tratamiento y con ellos se fertilizó tres grupos de ovas. Inmediatamente se agregó $0,01 \mathrm{ml}$ del pool de semen y se realizó la mezcla de los gametos manualmente. Luego de $5 \mathrm{~min}$ se agregó agua a los gametos y se procedió a eliminar los restos de semen y otros contaminantes. Posteriormente, se dejó hidratar las ovas por un tiempo de una hora. La incubación se realizó a $10,3 \pm 1,2^{\circ} \mathrm{C}$ en un sistema de flujo abierto. Después de 10 días 
de incubación se determinó el porcentaje de fecundación utilizando una solución de ácido acético al $10 \%$ en la que se introdujo una muestra de 20 ovas de cada réplica. Todas aquellas ovas en las que se observó el tubo neural, fueron consideradas fecundadas. La densidad espermática del pool de semen se determinó en cuatro grupos mediante recuento con cámara de Neubauer según metodología descrita por Oppenheim (1973).

Los promedios de motilidad y fertilidad fueron contrastados mediante ANDEVA a un nivel de significancia de $p \leq 0,001$ y 0,05 , respectivamente.

\section{RESULTADOS}

\section{Motilidad}

La densidad espermática obtenida en el pool de semen fue de $12,7 \pm 1,6 \times 10^{9}$ espermatozoides por $\mathrm{ml}$. El menor tiempo de motilidad se registró con la solución control $(80,8 \pm 12,5 \mathrm{~s})$ y el mayor valor $(165,0 \pm 18,8 \mathrm{~s})$ en el tratamiento de $10 \mathrm{mM}$ de cafeína, observándose un descenso del tiempo de motilidad con el tratamiento de $20 \mathrm{mM}$ (Fig. 1). Las diferencias fueron significativas entre el control y los tratamientos 2,3 y 4 . Se determinó además, diferencias significativas entre los tratamiento 1-3 y 2-3.

\section{Fertilidad}

La densidad espermática utilizada para el proceso de fertilización fue de 423.333 espermatozoides por ova en todos los tratamientos y controles. Los porcentajes de fecundación obtenidos fueron de 81,66 \pm $5,7 \%$ con el control; de $70,0 \pm 13,2 \%$ con $\mathrm{T}_{1}$; de $91,6 \pm 5,0 \%$ con el $\mathrm{T}_{2}$; de $95,0 \pm 5,0 \%$ con el $\mathrm{T}_{3}$ y $78,3 \pm 10,4 \%$ con el $\mathrm{T}_{4}$ (Fig. 2 ). Las diferencias registradas fueron estadísticamente significativas entre $T_{1}$ y $T_{3}$ para $p<0,05$.

\section{DISCUSIÓN}

El espermatozoide de salmónidos se caracteriza por estar inmóvil en el fluido seminal y sólo al tomar contacto con el agua y el $\mathrm{Ca}^{++}$que contiene, se activa su motilidad sólo por aproximadamente 30s (Billard, 1990; Perchec et al., 1993; Darszon et al., 1999). Esto ha motivado el uso de soluciones activadoras que prolongan la motilidad espermática y además, mejoran su fertilidad protegiendo la célula de un choque osmótico y mejorando su capacidad de generación de energía (Baynes et al., 1981). Los resultados obtenidos en esta investigación, demuestran la capacidad de la cafeína de prolongar la motilidad espermática en truchas, ya que los valores de actividad registrados son muy superiores a los informados por la literatura

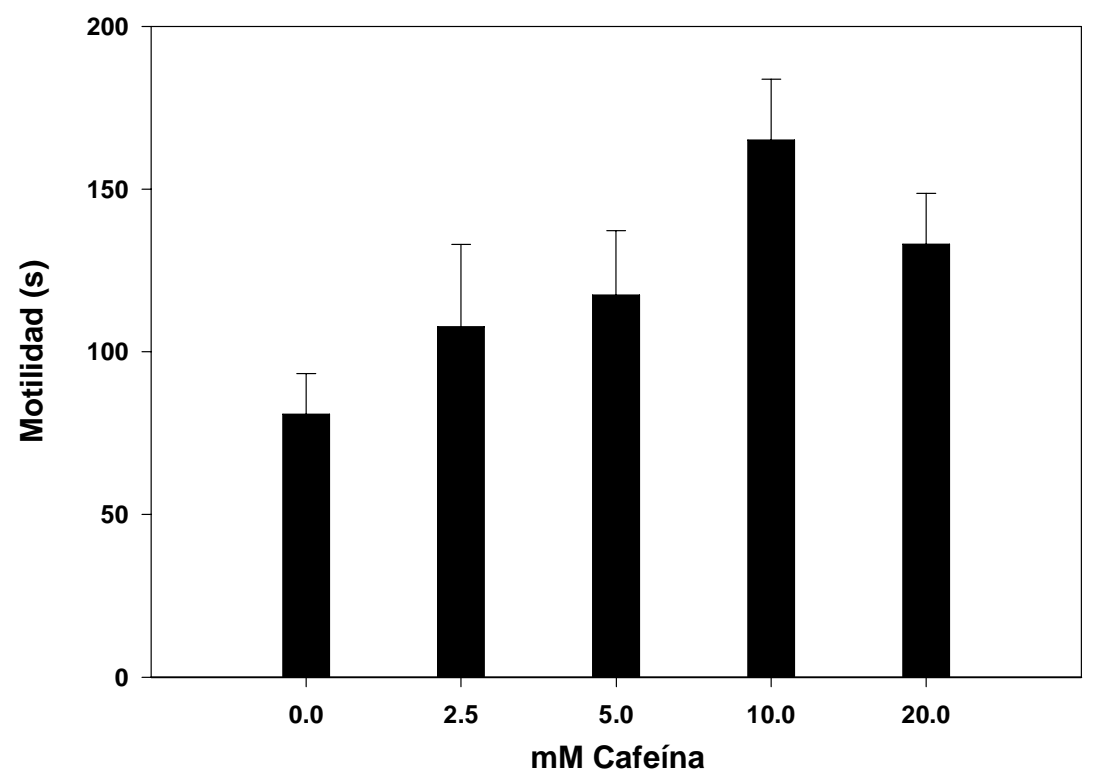

Fig. 1: Valores (promedios \pm Desviación estándar) de la motilidad (s) del espermatozoide de trucha arcoiris, obtenidos con diferentes concentraciones de cafeína. 


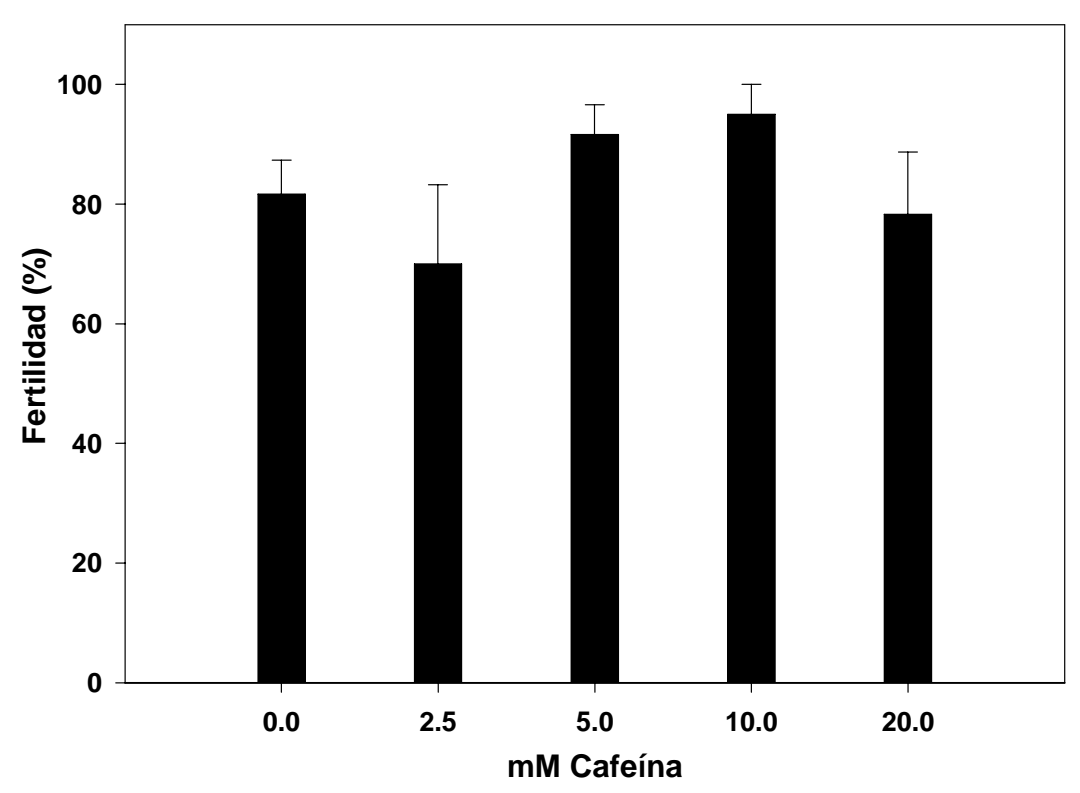

Fig. 2: Valores (promedios \pm Desviación estándar) de la fertilidad (\%) del espermatozoide de trucha arcoiris, obtenidos con diferentes concentraciones de cafeína.

para salmónidos (Terner, 1986; Billard y Cosson 1989, y Billard, 1990). Además, corroboran lo informado por Terner (1986) y Valdebenito y Ratto (1995) quienes señalan que la motilidad del espermatozoide de salmónidos puede ser intensificada y prolongada agregando cafeína en el diluyente espermático. Este efecto se debe a que la cafeína inhibe a la enzima fosfodiesterasa permitiendo la acumulación de nucleótidos cíclicos, especialmente del AMPc intracelular, provocando un aumento de la actividad flagelar (Aitken et al., 1983; Morisawa, 1994).

La adición de cafeína durante la fertilización, mejoró significativamente la duración de la motilidad, pero no la fertilidad del semen, esto coincide con lo reportado por Robles et al. (2003) quienes informan que el semen criopreservado de trucha arcoiris activado con soluciones que contenían metilxantinas, usualmente incrementaron la motilidad $y$ fertilidad, pero no significativamente con respecto a los controles. Ciereszko et al. (1996) determina que dosis de $5 \mathrm{mM}$ de teofilinas evitan la reducción de la motilidad espermática en esturiones hasta cinco minutos después de la activación.

Aas et al. (1991), determinan una correlación de 0,52 entre fertilidad y densidad espermática, razón por la cual en la presente investigación se fertilizó con una densidad espermática de 423.333 espermatozoides por ova. Valor menor a los 620.000 espermatozoides por ova utilizados por Aas et al. (1991) en salmón Atlántico, pero superior al señalado por Billard (1990) para la fertilización artificial en salmónidos, quien recomienda densidades de entre 100.000 y 300.000 espermatozoides por ova.

El incremento significativo de la motilidad espermática que produce la cafeína debiera reflejarse en un incremento de la fertilidad para justificar su incorporación en los activadores espermáticos de salmónidos, por lo que se deben realizar nuevas investigaciones que lo confirmen.

\section{CONCLUSIONES}

La cafeína a una concentración de $10 \mathrm{mM}$ en el medio isotónico de fertilización de ovas de trucha arcoiris, permite incrementar significativamente la motilidad espermática en trucha arcoiris.

\section{AGRADECIMIENTOS}

Investigación financiada por el Proyecto DIUCT 99.3.01.

\section{REFERENCIAS}

Aas G.H., T. Refstie y D. Gjerde, Evaluation of milt quality of Atlantic salmon. Aquaculture, 95:125-132 (1991).

Información Tecnológica - Vol. 18 No 2 - 2007 
Aitken, R.J., F. Best, D.W. Richardson, R. Schats y G. Simm, Influence of caffeine on movement characteristics, fertilizing capacity and ability to penetrate cervical mucus of human spermatozoa. Journal Reproduction and Fertility. 67:19-27 (1983).

Baynes, S.M., A.P. Scott y A.P. Dawson, Rainbow trout, Salmo gairdneri Richardson, spermatozoa: effects of cations and $\mathrm{pH}$ on motility. Journal of Fish Biology, 19:259-267 (1981).

Billard, R., Artificial insemination in fish. In Marshall's Physiology of reproduction. G.E. Lamming (Ed.), 2: 870-888 (1990).

Billard, R. y M.P. Cosson, Sperm motility in rainbowtrout, Parasalmo mykiss; effect of $\mathrm{pH}$ and temperature. Les Colloques de I'INRA, 44:161-167 (1988).

Billard, R. y M. Cosson, Measurement of sperm motility in trout and carp. In Aquaculture - A Biotechnology in Progress. N. de Pauw, E. Jaspers, H. Ackefors, N. Wilkins (Eds). European Aquaculture Society, Bredene, Gelgium: 499-503 (1989).

Billard, R. y B. Jalabert, L'insJmination artificielle de la truite Salmo gairdneri Richardson. Annales de Biologie Animale, Biochimie et Biophysique, 14:601-610 (1974).

Bouck, G.R. y J. Jacobson, Estimation of salmonid sperm concentration by microhematocrit technique. Transaction of the American Fisheries Society, 105:534-535 (1976).

Caylor, R.E., Biesiot P.M. y J.S. Franks, Culture of cobia (Rachycentron canadum): cryopreservation of sperm and induced spawning. Aquaculture 125:81-92 (1994).

Ciereszko, A., G.P. Toth, S. A Christ y K. Dabrowski, Effect of cryopreservation and theophylline on motility characteristics of lake sturgeon (Acipenser fulvescens) spermatozoa. Theriogenology 45(3):665-672 (1996).

Cosson, M.P., R. Billard, J.L. Gatti y R. Christen, Rapid and quantitative assessment of trout spermatozoa motility using stroboscopy. Aquaculture, 46:71-75 (1985).
Darszon, A., Labarca, P., Nishigaki, T. y F. Espinosa, lon channels in sperm physiology. Physiological Reviews 79(2): 481-510 (1999).

Ingram, M., Egg and milt. High Technology Broodstock Management. Clearwater Publishing Ltd., Surby, Port Erin, Isle of Man, British Isles, 111 (1985).

Levanduski, M.J. y J.G. Cloud, Rainbow trout (Salmo gairdneri) semen: effect of non motile sperm on fertility. Aquaculture, 75:171-179 (1988).

Morisawa, M, Cell signaling mechanisms for sperm motility. Zoological Science 11:647-662 (1994).

Oppenheim, I., Manual para técnicos de laboratorio. Editorial Panamericana. Bs. As. 188 (1973).

Perchec, G., J. Cosson, F. AndrJ. y R. Billard. Lamotilité des spermatozoïd de truite (Oncorhynchus mykiss) et de carpe (Cyprinus carpio). Journal of Applied Ichthyology, 9:129149 (1993).

Robles, V., E. Cabrita, S. Cuñado y P. Herráez, Sperm cryopreservation of sex-reversed rainbow trout (Oncorhynchus mykiss): parameters that affect its ability for freezing. Aquaculture 224(1-4):203-212 (2003).

Sánchez-Rodríguez, M. y R. Billard, Conservation de la motilité et du pouvoir fécondant du sperme de truite arc en el ciel maintenu a des temperatures voisines de $0^{\circ} \mathrm{C}$. Bulletin Francais de Pisciculture, (265):143152 (1977).

Stoss, J., Fish gamete preservation and spermatozoa physiology. In: W.S. Hoar, D.J. Randall and E.M. Donaldson (Eds), Fish Physiology, Vol. IXB., Academic Press, London, 305-350 (1983).

Terner, Ch., Evaluation of salmonid sperm motility for cryopreservation. The progressive Fish-Culturist 48:230-232 (1986).

Valdebenito I. y M. Ratto, Efecto de la cafeína en la motilidad y fertilidad del espermatozoide de trucha arco iris (Oncorhynchus mykiss). A. Silva y G. Merino (Eds.), Acuicultura en Latinoamérica. Asoc. Latinoamericana de Acuicultura. Coquimbo Chile: 370-373 (1996). 